\title{
Charged Higgs bosons in the NMSSM under current LHC constraints
}

\author{
Zhaoxia Heng, Lin Guo, Pengqiang Sun, Wei Wei \\ College of Physics and Materials Science, \\ Henan Normal University, Xinxiang 453007, China
}

\begin{abstract}
Charged Higgs boson is a crucial prediction of new physics beyond the SM. In this work, we perform a comprehensive scan over the parameter space of NMSSM considering various experimental constraints including the direct search limits from the $13 \mathrm{TeV}$ LHC, and consider the scenario that the next-to-lightest CP-even Higgs boson is SM-like. We find that the masses of charged Higgs bosons can be as light as $350 \mathrm{GeV}$, the lightest CP-even Higgs boson $h_{1}$ is predominantly singlet and can be as light as $48 \mathrm{GeV}$, and the lightest CP-odd Higgs boson $a_{1}$ is also singlet-dominated and can be as light as $82 \mathrm{GeV}$. The charged Higgs bosons mainly decay to $t \bar{b}$ or $\bar{t} b$, but the branching ratio of the exotic decays $H^{ \pm} \rightarrow W^{ \pm} h_{1}$ and $H^{ \pm} \rightarrow W^{ \pm} a_{1}$ can maximally reach to $20 \%$ and $11 \%$, respectively, which can be used to distinguish the NMSSM from MSSM. Such a heavy charged Higgs boson is unaccessible at the $13 \mathrm{TeV}$ LHC with a luminosity of $36.1 \mathrm{fb}^{-1}$ and its detection needs higher energy and/or higher luminosity.
\end{abstract}




\section{INTRODUCTION}

Both the ATLAS [1] and CMS [2] collaborations at the large Hadron Collider (LHC) announced the discovery of a Higgs boson with mass about $125 \mathrm{GeV}$ in 2012 , which implies that the standard model (SM) of elementary particles is fully established. However, many new physics models beyond the SM with extended Higgs sectors, such as the Minimal Supersymmetric Standard Model (MSSM) [3], can also accommodate a $125 \mathrm{GeV}$ Higgs boson. The MSSM consists of two Higgs doublet fields, which generate the masses of up- and downtype fermions. To realize a $125 \mathrm{GeV}$ Higgs, the MSSM needs large radiative corrections from the third generation squark loops [4 7], which makes the MSSM unnatural. And the MSSM also suffers from the $\mu$-problem. However, these problems can be remedied in the Next-toMSSM (NMSSM) [8], which extends the Higgs sector with an additional Higgs singlet field $\hat{S}$. The effective $\mu$-term can be generated when $\hat{S}$ acquires vacuum expectation value (vev). The coupling between the singlet and doublet Higgs fields can easily enhance the mass of the Higgs boson to be $125 \mathrm{GeV}$ without large radiative corrections [9 20]. In contrast to the MSSM, the NMSSM has richer Higgs spectrum, which contains three CP-even Higgs bosons, two CP-odd Higgs bosons and a pair of charged Higgs bosons $H^{ \pm}$. Needless to say, the discovery of extra Higgs bosons along with the SM-like Higgs boson will clearly confirm the existence of new physics beyond the SM.

Because of the different interactions and decay modes from neutral Higgs bosons, the studies of charged Higgs bosons have been received more and more attentions [21-25]. For charged Higgs bosons lighter than top-quark, they are mainly produced through top quark decay $t \rightarrow b H^{+}$, and primarily decay to $\tau \nu$ and $s c$. For charged Higgs bosons heavier than top-quark, they are produced at the LHC directly through the processes $p p \rightarrow t \bar{b} H^{ \pm}$, the pair production process $p p \rightarrow H^{+} H^{-}$and also the associated production with a neutral Higgs boson, then they may be searched via the relatively clean decay channel $H^{ \pm} \rightarrow \tau \nu$ [26, 27]. When kinematically allowed, the charged Higgs bosons decay to $t \bar{b}$ or $\bar{t} b$ dominantly, but it is challenging to reconstruct such events due to the large irreducible SM backgrounds [28]. Besides the conventional search channels, the Higgs exotic decay modes [29 31], such as $H^{ \pm} \rightarrow W^{ \pm} H / A(\mathrm{H} / \mathrm{A}$ denotes the neutral CP-even/CP-odd Higgs boson) [32, 33] have studied to provide complementary detection of charged Higgs bosons. So far, the void of any charged Higgs bosons signal events limits its production and decay in a model independent 
way, which in turn can be used to constrain the relevant parameter space.

In this work we examine the parameter space of NMSSM considering the experimental constraints from the $125 \mathrm{GeV}$ Higgs data, B-physics observables, the dark matter direct detection, and also the LHC direct search limits. We find that the charged Higgs bosons in the NMSSM can be as light as $350 \mathrm{GeV}$, and the exotic decay modes of the charged Higgs bosons are open, such as $H^{ \pm} \rightarrow W^{ \pm} h_{1}$ and $W^{ \pm} a_{1}\left(h_{1}\right.$ and $a_{1}$ are the lightest CP-even and CP-odd Higgs boson, respectively), which can be used to distinguish the NMSSM from MSSM. However, the LHC with the current luminosity has not found a charged Higgs boson. To detect the charged Higgs bosons in the mass range 350-500 GeV, higher luminosity and/or higher energy collider is needed [32, 34].

This work is organized as follows. In section II we briefly describe the NMSSM. In section III we first perform a comprehensive scan over the parameter space with the package NMSSMTools [35, 36], then we further constrain the parameter space using the LHC direct search limits, and discuss the future detection of charged Higgs bosons at the LHC. Finally, the conclusions are drawn in section IV.

\section{BASICS OF THE NMSSM}

As the most economic realization of supersymmetry, the MSSM consists of two Higgs doublets $\hat{H}_{u}$ and $\hat{H}_{d}$. Different from the MSSM, the NMSSM adds one extra Higgs singlet

field $\hat{S}$. The superpotential and soft breaking terms in the Higgs sector of the NMSSM are given by

$$
\begin{aligned}
W_{\mathrm{NMSSM}} & =W_{\mathrm{MSSM}}+\lambda \hat{H}_{u} \cdot \hat{H}_{d} \hat{S}+\frac{1}{3} \kappa \hat{S^{3}}, \\
V_{\mathrm{soft}}^{\mathrm{NMSSM}} & =m_{H_{u}}^{2}\left|H_{u}\right|^{2}+m_{H_{d}}^{2}\left|H_{d}\right|^{2}+m_{S}^{2}|S|^{2}+\left(A_{\lambda} \lambda S H_{u} \cdot H_{d}+\frac{A_{\kappa}}{3} \kappa S^{3}+\text { h.c. }\right) .
\end{aligned}
$$

with $W_{\text {MSSM }}$ being the superpotential of MSSM without $\mu$-term. At the tree level, the Higgs sector in the NMSSM consists of the following nine parameters:

$$
\lambda, \kappa, \tan \beta, \mu_{\mathrm{eff}}, A_{\lambda}, A_{\kappa}, m_{H_{u}}^{2}, m_{H_{d}}^{2}, m_{S}^{2}
$$

where $\tan \beta=v_{u} / v_{d}$ and $\mu_{\text {eff }}=\lambda v_{s}$ with $v_{u}, v_{d}, v_{s}$ denoting the vev of $H_{u}, H_{d}$ and $S$. The parameters $m_{H_{u}}^{2}, m_{H_{d}}^{2}, m_{S}^{2}$ can be determined by the minimization conditions of the scalar 
potential, so six independent parameters are left. Usually, the following six parameters are chosen as input parameters,

$$
\lambda, \kappa, \tan \beta, \mu_{\mathrm{eff}}, M_{A}^{2}=\frac{2 \mu_{\mathrm{eff}}}{\sin 2 \beta}\left(A_{\lambda}+\kappa v_{s}\right), A_{\kappa} .
$$

Assume $H_{1}=\cos \beta H_{u}-\varepsilon \sin \beta H_{d}^{*}, H_{2}=\sin \beta H_{u}+\varepsilon \cos \beta H_{d}^{*}$ with $\varepsilon$ being two-dimensional anti-symmetric matrix with off-diagonal elements of $(1,-1)$, the Higgs fields in the NMSSM can be written as [18, 37]:

$$
H_{1}=\left(\begin{array}{c}
H^{+} \\
\frac{S_{1}+i P_{1}}{\sqrt{2}}
\end{array}\right), \quad H_{2}=\left(\begin{array}{c}
G^{+} \\
v+\frac{S_{2}+i G^{0}}{\sqrt{2}}
\end{array}\right), \quad H_{3}=s+\frac{1}{\sqrt{2}}\left(S_{3}+i P_{2}\right),
$$

where $G^{+}$and $G^{0}$ are Goldstone bosons. Obviously, the field $H_{2}$ corresponds to the SM Higgs field. At tree-level, the mass matrices $\mathcal{M}_{S}^{2}$ (under the basis $\left(S_{1}, S_{2}, S_{3}\right)$ ) and $\mathcal{M}_{P}^{2}$ (under the basis $\left(P_{1}, P_{2}\right)$ ) are given by, respectively,

$$
\begin{aligned}
& \left(\mathcal{M}_{S}^{2}\right)_{11}=M_{A}^{2}+\left(m_{Z}^{2}-\lambda^{2} v^{2}\right) \sin ^{2} 2 \beta \\
& \left(\mathcal{M}_{S}^{2}\right)_{12}=-\frac{1}{2}\left(m_{Z}^{2}-\lambda^{2} v^{2}\right) \sin 4 \beta \\
& \left(\mathcal{M}_{S}^{2}\right)_{13}=-\left(M_{A}^{2} \sin 2 \beta+\frac{2 \kappa \mu^{2}}{\lambda}\right) \frac{\lambda v}{\mu} \cos 2 \beta \\
& \left(\mathcal{M}_{S}^{2}\right)_{22}=m_{Z}^{2} \cos ^{2} 2 \beta+\lambda^{2} v^{2} \sin ^{2} 2 \beta \\
& \left(\mathcal{M}_{S}^{2}\right)_{23}=2 \lambda \mu v\left[1-\left(\frac{M_{A} \sin 2 \beta}{2 \mu}\right)^{2}-\frac{\kappa}{2 \lambda} \sin 2 \beta\right], \\
& \left(\mathcal{M}_{S}^{2}\right)_{33}=\frac{1}{4} \lambda^{2} v^{2}\left(\frac{M_{A} \sin 2 \beta}{\mu}\right)^{2}+\frac{\kappa \mu}{\lambda}\left(A_{\kappa}+\frac{4 \kappa \mu}{\lambda}\right)-\frac{1}{2} \lambda \kappa v^{2} \sin 2 \beta, \\
& \left(\mathcal{M}_{P}^{2}\right)_{11}=M_{A}^{2} \\
& \left(\mathcal{M}_{P}^{2}\right)_{12}=\frac{1}{2}\left(M_{A}^{2} \sin 2 \beta-6 \lambda \kappa v_{s}^{2}\right) \frac{v}{v_{s}} \\
& \left(\mathcal{M}_{P}^{2}\right)_{22}=\frac{1}{4}\left(M_{A}^{2} \sin 2 \beta+6 \lambda \kappa v_{s}^{2}\right)\left(\frac{v}{v_{s}}\right)^{2} \sin 2 \beta-3 \kappa v_{s} A_{\kappa} .
\end{aligned}
$$

Using the rotation matrices $U^{S}$ and $U^{P}$ to diagonalize the matrices $\mathcal{M}_{S}^{2}$ and $\mathcal{M}_{P}^{2}$, respectively, the CP-even and CP-odd Higgs mass eigenstate can be obtained by $h_{i}=\sum_{j=1}^{3} U_{i j}^{S} S_{j}$, $a_{i}=\sum_{j=1}^{2} U_{i j}^{P} P_{j}$. We call the scalar $h_{i}$ with largest $S_{2}$ component being the SM-like Higgs boson $h$ and take $m_{h_{1}}<m_{h_{2}}<m_{h_{3}}$ and $m_{a_{1}}<m_{a_{2}}$. The element of mass matrix $\mathcal{M}_{22}^{2}$ indicates that the mass of SM-like Higgs boson receives an additional contribution $\lambda^{2} v^{2} \sin ^{2} 2 \beta$ in contrast with that of MSSM. Furthermore, the $\left(S_{2}, S_{3}\right)$ mixing can also raise the mass of SM-like Higgs boson if $\left(\mathcal{M}_{S}^{2}\right)_{22}>\left(\mathcal{M}_{S}^{2}\right)_{33}$. This case corresponds to the next-to-lightest 
CP-even Higgs boson being SM-like. In this work, we only consider the scenario with $h_{2}$ being SM-like Higgs boson.

The masses of charged bosons $H^{ \pm}$at tree-level can be obtained as [38, 39]

$$
m_{H^{ \pm}}^{2}=M_{A}^{2}+m_{W}^{2}-\lambda^{2} v^{2}
$$

and the couplings of charged Higgs boson with the third generation fermions are as follows,

$$
\begin{aligned}
g_{H^{-} t \bar{b}} & =\frac{g_{2}}{\sqrt{2} m_{W}}\left(m_{b} \tan \beta P_{L}+m_{t} \cot \beta P_{R}\right) \\
g_{H^{-}-\bar{\tau} \nu} & =\frac{g_{2}}{\sqrt{2} m_{W}} m_{\tau} \tan \beta P_{L} .
\end{aligned}
$$

In the neutralino sector, the gauginos $\tilde{B}$ and $\tilde{W}^{0}$ mix with the neutral Higgsinos $\tilde{H}_{u}^{0}, \tilde{H}_{d}^{0}$ and singlino $\tilde{S}$ to form a symmetric $5 \times 5$ mass matrix $\mathcal{M}_{0}$. In the basis $\psi=\left(-i \tilde{B},-i \tilde{W}^{0}, \tilde{H}_{d}^{0}, \tilde{H}_{u}^{0}, \tilde{S}\right)$ the matrix $\mathcal{M}_{0}$ is given by [8]

$$
\mathcal{M}_{0}=\left(\begin{array}{ccccc}
M_{1} & 0 & -\frac{g_{1} v_{d}}{\sqrt{2}} & \frac{g_{1} v_{u}}{\sqrt{2}} & 0 \\
& M_{2} & \frac{g_{2} v_{d}}{\sqrt{2}} & -\frac{g_{2} v_{u}}{\sqrt{2}} & 0 \\
& & 0 & -\mu_{\mathrm{eff}} & -\lambda v_{u} \\
& & & 0 & -\lambda v_{d} \\
& & & & 2 \kappa v_{s}
\end{array}\right)
$$

with $M_{1}\left(M_{2}\right)$ being Bino (Wino) mass term, and $g_{1}, g_{2}$ being SM gauge couplings. Using the rotation matrix $N$ to diagonalize the matrix $\mathcal{M}_{0}$, the mass eigenstates $\tilde{\chi}_{i}^{0}$ are written as

$\tilde{\chi}_{i}^{0}=\sum_{j=1}^{5} N_{i j} \psi_{j}$, and the masses of neutralino are arranged in ascending order.

As in the MSSM, the charged gauginos $\tilde{W}^{+}, \tilde{W}^{-}$mix with the charged Higgsinos $\tilde{H}_{u}^{+}$, $\tilde{H}_{d}^{-}$to form two mass eigenstates called charginos $\tilde{\chi}_{i}^{ \pm}(i=1,2)$ with $m_{\tilde{\chi}_{1}^{ \pm}}<m_{\tilde{\chi}_{2}^{ \pm}}$.

\section{CALCULATIONS AND NUMERICAL RESULTS}

\section{A. Scan strategies and preliminary results}

We use the package NMSSMTools [35, 36] to obtain the particle spectrum, decay branching ratios of Higgs bosons and relevant couplings. Firstly we fix the gluino mass to be 1900 $\mathrm{GeV}$ and the soft breaking parameters in the first two generation squark to be $2 \mathrm{TeV}$. We assume the soft breaking parameters for the left- and right-handed states in the slepton 
sector to be $m_{\tilde{l}}=350 \mathrm{GeV}$ and $A_{\tau}=A_{e}=A_{\mu}=1500 \mathrm{GeV}$. The absence of a Landau pole below the GUT scale implies that $\lambda, \kappa \leq 0.7$. The lower limit on chargino masses from LEP is $103.5 \mathrm{GeV}$ and the naturalness usually requires a low value of $\mu$, so we choose 100 $\mathrm{GeV} \leq \mu \leq 500 \mathrm{GeV}$. Considering the constraints from LHC search for electroweakinos, we choose $M_{2} \leq 1 \mathrm{TeV}$. In this work, we focus on searching for a light charged Higgs boson, so we require $M_{A} \leq 500 \mathrm{GeV}$. Then we perform a comprehensive scan over the following parameter regions:

$$
\begin{aligned}
& 0.001<\lambda, \kappa \leq 0.7, \quad 1.5 \leq \tan \beta \leq 60 \\
& 100 \mathrm{GeV} \leq \mu \leq 500 \mathrm{GeV}, \quad 0 \leq M_{P} \leq 1000 \mathrm{GeV} \\
& 50 \mathrm{GeV} \leq M_{1} \leq 400 \mathrm{GeV}, \quad 50 \mathrm{GeV} \leq M_{2} \leq 1000 \mathrm{GeV} \\
& 300 \mathrm{GeV} \leq M_{Q_{3}}, M_{U_{3}}\left(M_{D_{3}}\right) \leq 2000 \mathrm{GeV}, \\
& -4000 \mathrm{GeV} \leq A_{t}=A_{b} \leq 4000 \mathrm{GeV}, \quad 100 \mathrm{GeV} \leq M_{A} \leq 500 \mathrm{GeV}
\end{aligned}
$$

where $M_{P}$ is the singlet diagonal element of CP-odd Higgs mass matrix.

We pick up the samples that satisfy the following constraints:

- The direct mass bounds on Higgs bosons and sparticles from LEP and Tevatron experiments.

- The constraints from B-physics observables such as the branching ratio of processes $B_{s} \rightarrow \mu^{+} \mu^{-}, B \rightarrow X_{s} \gamma$ and $B_{u} \rightarrow \tau \nu$. We require their theoretical predications is within $2 \sigma$ range of the corresponding experimental values.

- The constraints from the dark matter relic density with $0.1068<\Omega h^{2}<0.13057$ consistent with the Planck measurement [40]. Because of the existence of blind spots [41, 42] for neutralino dark matter in the NMSSM, we do not consider the constraints from dark matter direct detection experiments, such as LUX and XENON-1T experiments.

- The constraints from Higgs data. We assume the next-to-lightest CP-even Higgs boson being SM-like and satisfying $122 \mathrm{GeV} \leq m_{h_{2}} \leq 128 \mathrm{GeV}$. We consider the constraints from LEP, Tevatron and LHC on the direct searches for neutral and charged Higgs bosons using the package HiggsBounds [43, 44] and perform the fit for the $125 \mathrm{GeV}$ Higgs data using the package HiggsSignals [45-47]. 
In Fig 1 and Fig, 2 we display the surviving samples in the $U_{13}^{S}-m_{h_{1}}, U_{12}^{P}-m_{a_{1}}$ planes, respectively. Fig 1 shows that $h_{1}$ is predominantly singlet and can be as light as $48 \mathrm{GeV}$. Fig 2 shows that $a_{1}$ is also dominated by singlet component and can be as light as $82 \mathrm{GeV}$. In Fig 3 we display the surviving samples in the $M_{A}-m_{H^{ \pm}}$plane. The figure shows that the masses of charged Higgs bosons can be as light as $350 \mathrm{GeV}$ and are highly correlated with $M_{A}$, which can be seen clearly from Eq.(15). The mass upper bound of charged Higgs bosons is due to the requirement of $M_{A} \leq 500 \mathrm{GeV}$.

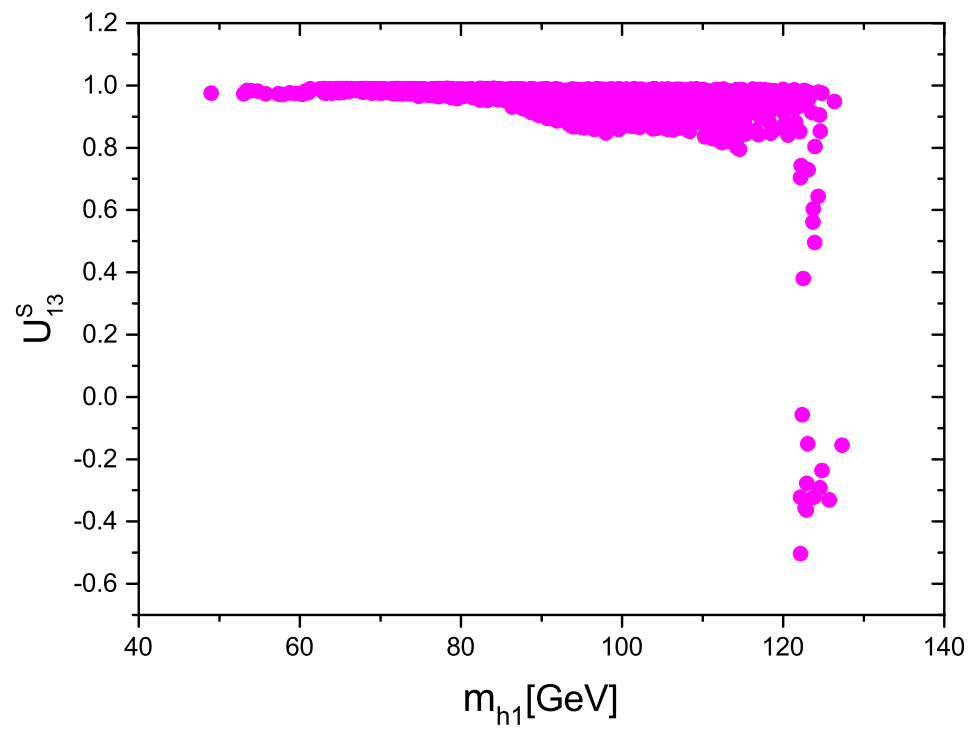

FIG. 1: Surviving samples in the $U_{13}^{S}-m_{h_{1}}$ plane.

In Fig, 4 we show the different decay channels of charged Higgs bosons in the NMSSM. From the figure, we can see that the dominant decay channel is $H^{+} \rightarrow t \bar{b}$, which varies from $71 \%$ to $28 \%$. Although the decay channel $H^{ \pm} \rightarrow \tau^{ \pm} \nu_{\tau}$ may be dominant for light charged Higgs bosons, in our case it is rather small. Firstly it is because the $H^{ \pm} \tau \nu_{\tau}$ coupling is suppressed for small $\tan \beta$, which can be seen from Eq.(16). Secondly, some exotic decay channels are open, such as $H^{ \pm} \rightarrow W^{ \pm} h_{1}, H^{ \pm} \rightarrow W^{ \pm} a_{1}$ and $H^{ \pm} \rightarrow \tilde{\chi}_{1}^{ \pm} \tilde{\chi}_{i}^{0}$. The figure shows that $\operatorname{Br}\left(H^{ \pm} \rightarrow W^{ \pm} h_{1}\right)$ and $\operatorname{Br}\left(H^{ \pm} \rightarrow W^{ \pm} a_{1}\right)$ can maximally reach to $20 \%$ and $11 \%$, respectively, and the branching ratio of $H^{ \pm} \rightarrow \tilde{\chi}_{1}^{ \pm} \tilde{\chi}_{1}^{0}$ can reach to about $22 \%$. As is well known, the branching ratio of the decay $H^{ \pm} \rightarrow W^{ \pm} h_{1} / a_{1}$ is strongly dependent on the mass of $h_{1} / a_{1}$ and the coupling $W^{ \pm} H^{\mp} h_{1} / a_{1}$, which is directly proportional to the doublet component of $h_{1} / a_{1}$. Although $h_{1}$ and $a_{1}$ are dominated by singlet component, they 


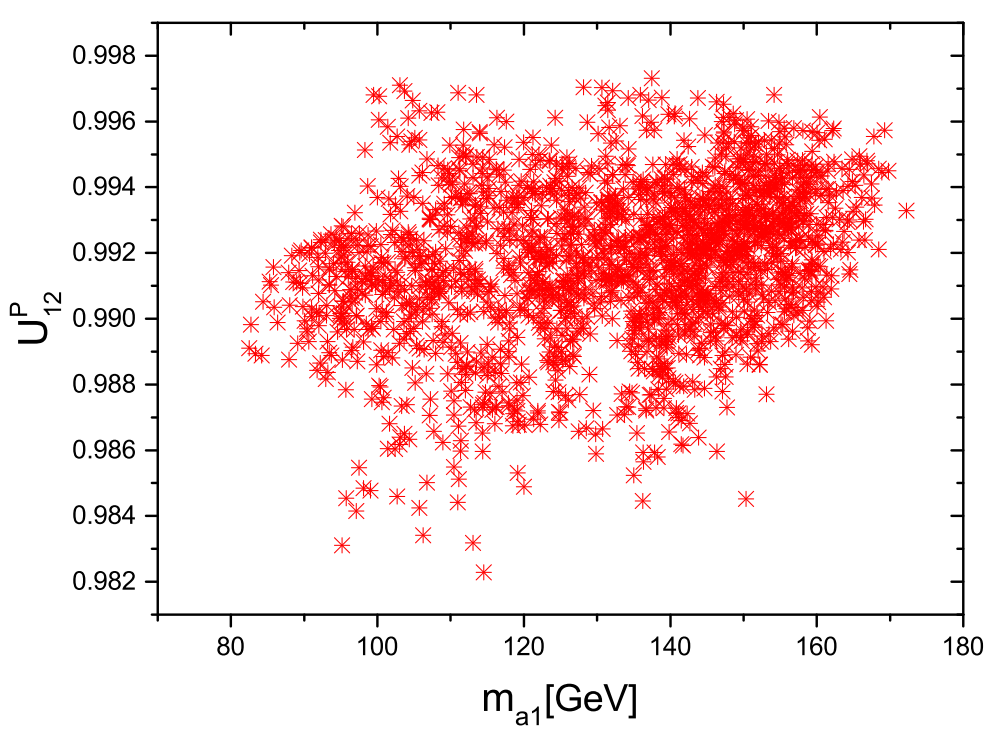

FIG. 2: Surviving samples in the $U_{12}^{P}-m_{a_{1}}$ plane.

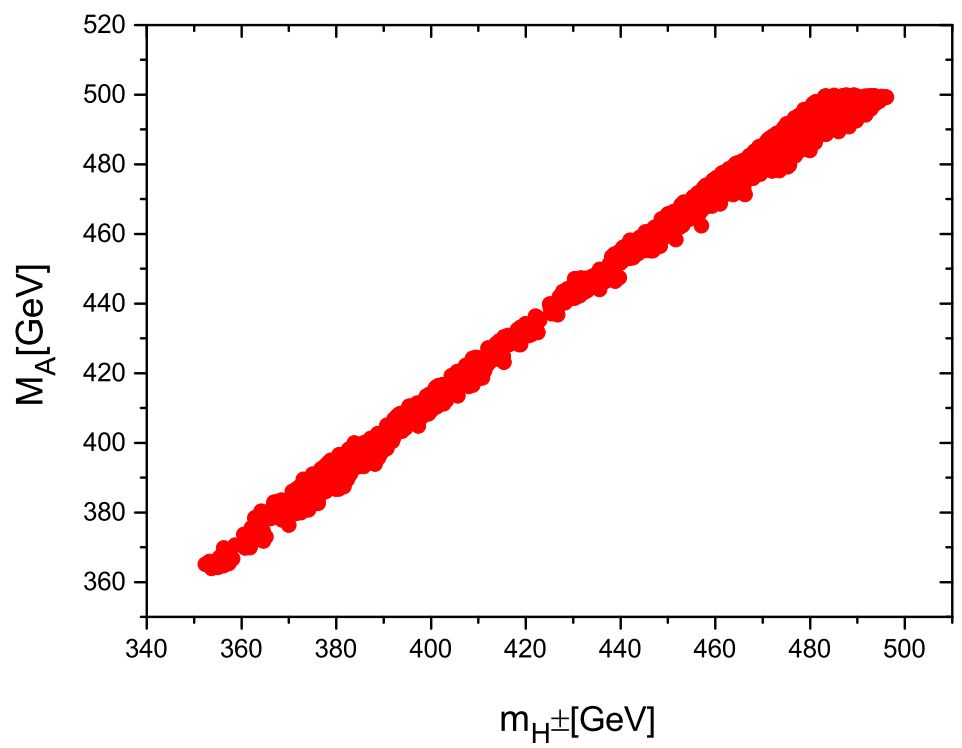

FIG. 3: Surviving samples in the $M_{A}-m_{H^{ \pm}}$plane.

can be very light (see Fig,1 and Fig,2), therefore the branching ratio of $H^{ \pm} \rightarrow W^{ \pm} h_{1} / a_{1}$ can be sizeable. In the MSSM, the relationship $m_{H^{ \pm}}^{2}=M_{A}^{2}+m_{W}^{2}$ ensures that the decay $H^{ \pm} \rightarrow W^{ \pm} A$ is strongly suppressed in most of the parameter space. Therefore, the detection of the decay channel $H^{ \pm} \rightarrow W^{ \pm} h_{1} / a_{1}$ can be used to distinguish the NMSSM from MSSM. 

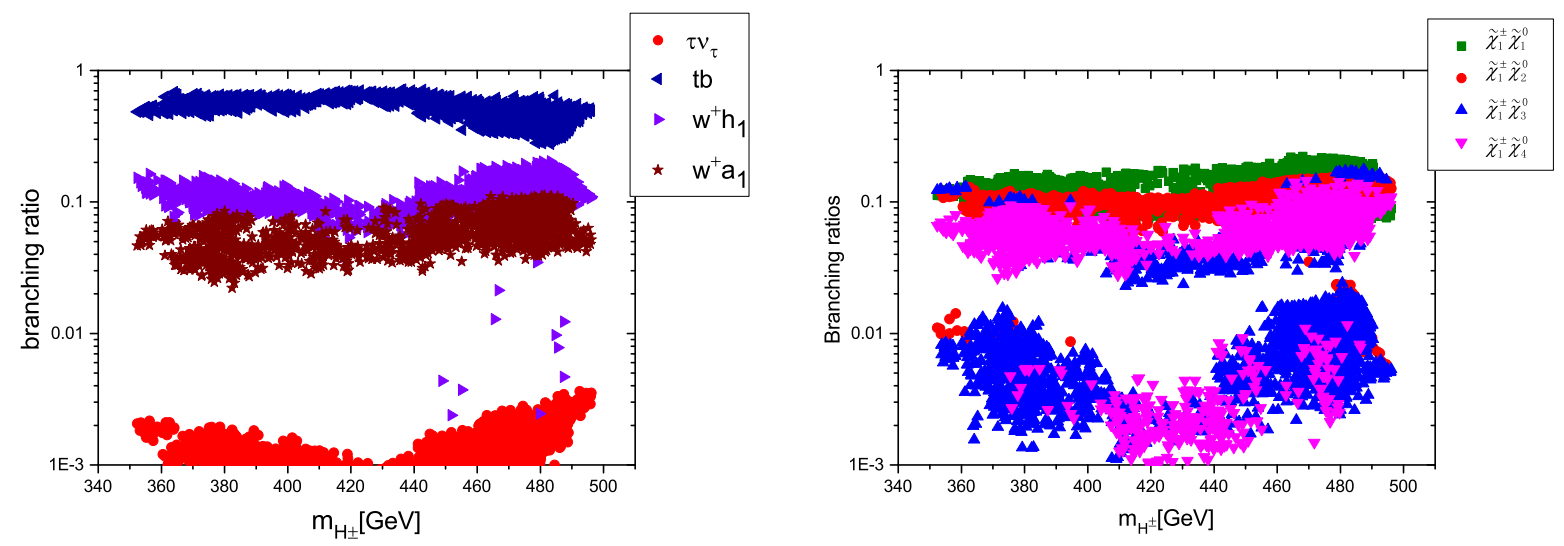

FIG. 4: Branching ratios of different decay channels of charged Higgs bosons versus $m_{H^{ \pm}}$.

\section{B. The LHC direct search limits}

Both the ATLAS and CMS collaborations have searched for the charged Higgs bosons via the production process $t \rightarrow b H^{+}$or $p p \rightarrow t b H^{ \pm}$, subsequently charged Higgs bosons decay to $\tau \nu$ [26, 27]. With $\mathcal{L}=36.1 \mathrm{fb}^{-1}$ at $13 \mathrm{TeV}$, the observed $95 \%$ C.L. upper limits from the ATLAS [26] on the production cross section of $H^{ \pm}$times $\operatorname{Br}\left(H^{ \pm} \rightarrow \tau^{ \pm} \nu\right)$ is between 4.2 $\mathrm{pb}$ and $2.5 \mathrm{fb}$ for $90 \mathrm{GeV} \lesssim \mathrm{m}_{\mathrm{H}^{ \pm}} \lesssim 2000 \mathrm{GeV}$. Interpreting in the hMSSM scenario of the MSSM, charged Higgs bosons lighter than $160 \mathrm{GeV}$ are excluded for all $\tan \beta$ values, and charged Higgs bosons lighter than $1100 \mathrm{GeV}$ are excluded when $\tan \beta=60$. The CMS gave the similar results [27]. The ATLAS also searched for charged Higgs bosons via the process $p p \rightarrow t b H^{ \pm}$and then decaying to $t b[28]$. With $\mathcal{L}=36.1 \mathrm{fb}^{-1}$ at $13 \mathrm{TeV}$, the observed $95 \%$ C.L. upper limits on $\sigma\left(p p \rightarrow t b H^{ \pm}\right) \times B r\left(H^{ \pm} \rightarrow t b\right)$ is in the range 2.9-0.07pb for $200 \mathrm{GeV} \lesssim \mathrm{m}_{\mathrm{H}^{ \pm}} \lesssim 2000 \mathrm{GeV}$. In the hMSSM scenario, $0.5<\tan \beta<1.95$ is excluded for $200 \mathrm{GeV} \lesssim \mathrm{m}_{\mathrm{H}^{ \pm}} \lesssim 965 \mathrm{GeV}$.

To compare with the LHC direct search limits, we calculate the production cross section of the process $p p \rightarrow t b H^{ \pm}$times $B r\left(H^{ \pm} \rightarrow \tau^{ \pm} \nu\right)$ or $\operatorname{Br}\left(H^{ \pm} \rightarrow t b\right)$ at $\sqrt{s}=13 \mathrm{TeV}$, and show the results in Fig,5 and Fig.6, which also shows the observed 95\% C.L. exclusion limits on $\sigma \times B r$ from ATLAS [26, 28] and CMS [27] with integrated luminosity of $36.1 \mathrm{fb}^{-1}$ at $\sqrt{s}$ $=13 \mathrm{TeV}$. The figure indicates that the $\sigma \times B r$ of the surviving samples are less than the observed 95\% C.L. exclusion limits from the ATLAS and CMS.

Due to the large irreducible SM backgrounds, we also consider the LHC direct search 


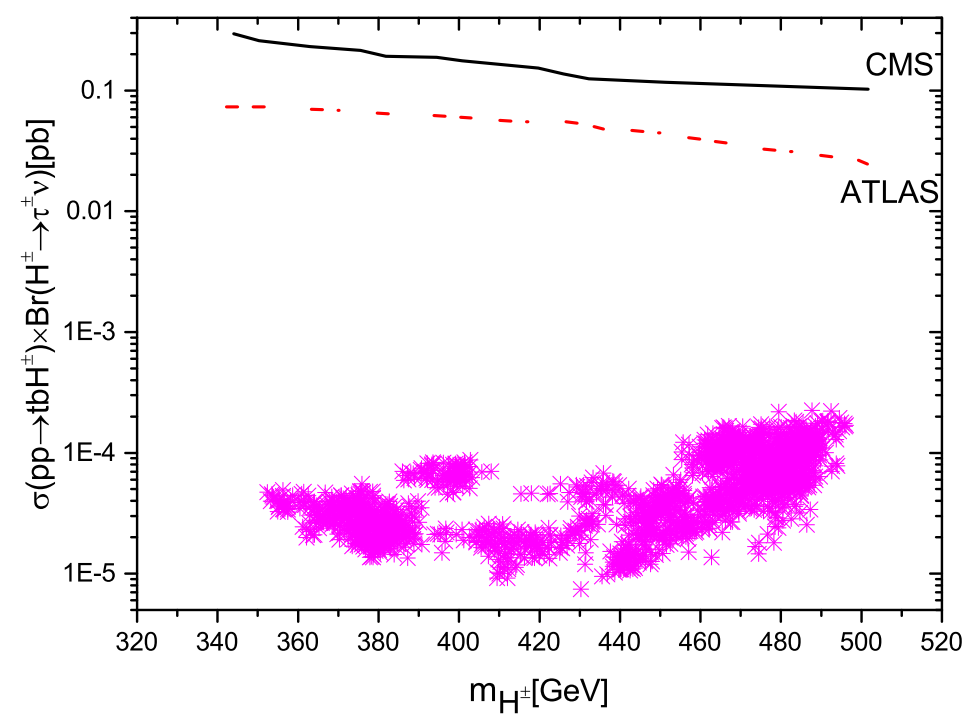

FIG. 5: Surviving samples in the $\sigma\left(p p \rightarrow t b H^{ \pm}\right) \times B r\left(H^{ \pm} \rightarrow \tau^{ \pm} \nu\right)-m_{H^{ \pm}}$plane. The observed 95\% C.L. exclusion limits on $\sigma \times B r$ from ATLAS and CMS with integrated luminosity of 36.1 $\mathrm{fb}^{-1}$ at $\sqrt{s}=13 \mathrm{TeV}$ are also shown in the figure.

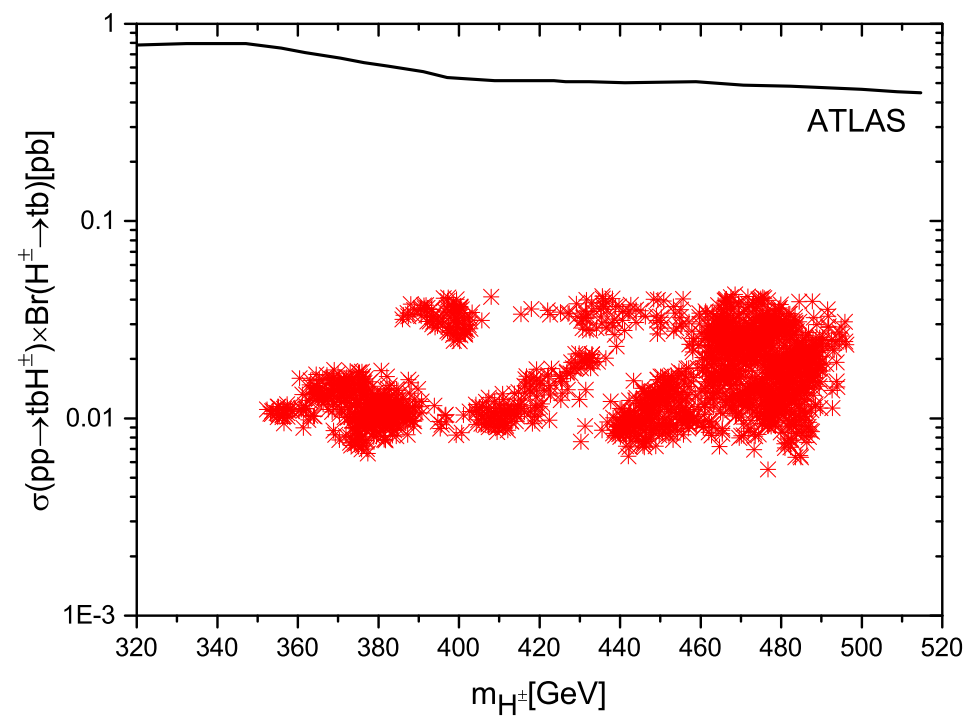

FIG. 6: Surviving samples in the $\sigma\left(p p \rightarrow t b H^{ \pm}\right) \times B r\left(H^{ \pm} \rightarrow t b\right)-m_{H^{ \pm}}$plane (right). The observed 95\% C.L. exclusion limits on $\sigma \times B r$ from ATLAS with integrated luminosity of $36.1 \mathrm{fb}^{-1}$ at $\sqrt{s}$ $=13 \mathrm{TeV}$ are also shown in the figure. 
limits for the charged Higgs bosons produced through the electroweak processes [48]:

$$
\begin{aligned}
& p p \rightarrow W^{ \pm *} \rightarrow H^{ \pm} h_{i}(i=1,2,3) \\
& p p \rightarrow W^{ \pm *} \rightarrow H^{ \pm} a_{j}(j=1,2) \\
& p p \rightarrow Z^{*} / \gamma^{*} \rightarrow H^{+} H^{-} .
\end{aligned}
$$

and consider the decay channel of charged Higgs bosons to $\tau^{ \pm} \nu$ or $W^{ \pm} h_{1} / a_{1}$. For the heavier neutral Higgs bosons $h_{3}$ and $a_{2}$, their dominant decay channels are $h_{3} \rightarrow a_{1} Z, a_{2} \rightarrow h_{1} Z$. For the lighter neutral Higgs bosons $h_{1}$ and $a_{1}$, they mainly decay to $b \bar{b}$ with branching ratio close to $90 \%$, and subsequently decay to $\tau^{+} \tau^{-}$with branching ratio close to $10 \%$. In order to reduce the irreducible background, we consider the decay mode of $h_{1} / a_{1} \rightarrow \tau^{+} \tau^{-}$. The cross sections of processes $p p \rightarrow W^{ \pm *} \rightarrow H^{ \pm} h_{1} / a_{1}$ are strongly correlated with the coupling $W^{ \pm} H^{\mp} h_{1} / a_{1}$, which is directly proportional to the doublet component of $h_{1} / a_{1}$. Since $h_{1} / a_{1}$ are singlet dominated, the cross sections of processes $p p \rightarrow W^{ \pm *} \rightarrow H^{ \pm} h_{1} / a_{1}$ are suppressed. So we consider the following final states,

$$
\begin{gathered}
p p \rightarrow W^{ \pm *} \rightarrow H^{ \pm} h_{3} / a_{2} \rightarrow 3 \tau+\nu_{\tau}+Z \text { or } 4 \tau+Z+W^{ \pm}, \\
p p \rightarrow Z^{*} / \gamma^{*} \rightarrow H^{+} H^{-} \rightarrow 2 \tau+\nu_{\tau} \text { or } 4 \tau+W^{+} W^{-} \\
\text {or } 3 \tau+\nu_{\tau}+W^{+} / W^{-} .
\end{gathered}
$$

We generate the parton level signal events using MG5_aMC-2.4.3 [49] with PYTHIA6 [50] performing parton showering and hadronization, then we use CheckMATE-2.0.7 [51] with all the analysis at $13 \mathrm{TeV}$ LHC to perform simulations. For most of the surviving samples, $R<1$, where $R=\max _{i}\left\{R_{i}\right\}$ with $R_{i}$ being the limit for each searching analysis by ATLAS or CMS. $R_{i}=\max _{j}\left\{R_{i, j}\right\}$ with $j$ standing for each signal region at one analysis, and $R_{i, j}=\frac{S}{S_{o b s}^{95}}$ with $S$ being predicted events number of the model and $S_{o b s}^{95}$ being upper limit of events number at $95 \%$ confidence level.

Combined the LHC direct search limits discussed above, the charged Higgs bosons can be as light as $350 \mathrm{GeV}$ in the NMSSM.

\section{The future detection of charged Higgs bosons}

When charged Higgs bosons heavier than top quark, it dominantly decay to $t \bar{b}$ or $\bar{t} b$. But such signal events are overwhelmed by the large SM background. And the branching 
ratio of the clean decay channel $H^{ \pm} \rightarrow \tau^{ \pm} \nu$ is rather small. Therefore, it is challenging to search for charged Higgs bosons through the conventional search channels. Ref.[32] studied the exotic decay channel of charged Higgs bosons $H^{ \pm} \rightarrow A W^{ \pm}$in two Higgs doublet model $(2 \mathrm{HDM})$. For $m_{A}=70 \mathrm{GeV}$ with small and large $\tan \beta$, a charged Higgs boson lighter than $400 \mathrm{GeV}$ may be discovered with $\mathcal{L}=300 \mathrm{fb}^{-1}$ at the LHC. In the $m_{H^{ \pm}}-\tan \beta$ plane, the charged Higgs bosons with masses extended to $600 \mathrm{GeV}$ can be excluded at the $95 \%$ C.L.. Adopting the multivariate analysis technique to improve the signal sensitivity, Ref.[34] studied the signature of a heavier charged Higgs boson for the decay mode $H^{+} \rightarrow t \bar{b}$ with both the hadronic and leptonic final states. For $\tan \beta=3$, the charged Higgs bosons in 2HDM with mass between 300 and $600 \mathrm{GeV}$ may be observable at the $\mathrm{LHC}$ with $\mathcal{L}=1000 \mathrm{fb}^{-1}$. Therefore, the future higher luminosity and/or higher energy colliders would be useful for the detection of charged Higgs bosons [52 54].

\section{CONCLUSION}

Due to the different peculiarity from the neutral Higgs bosons, the charged Higgs bosons have been received more and more attentions. The search for charged Higgs bosons would be a crucial signal of new physics beyond the SM. We perform a comprehensive scan over the parameter space of the NMSSM considering various experimental constraints including the direct search limits from the $13 \mathrm{TeV}$ LHC, and pick up the samples with the next-to-lightest CP-even Higgs boson being SM-like. We find that the masses of charged Higgs bosons are larger than $350 \mathrm{GeV}$, the lightest CP-even Higgs boson $h_{1}$ is predominantly singlet and can be as light as $48 \mathrm{GeV}$, and the lightest CP-odd Higgs boson $a_{1}$ is also singlet-dominated and can be as light as $82 \mathrm{GeV}$. We also discuss the different decay channels of the charged Higgs bosons. The charged Higgs bosons mainly decay to $t \bar{b}$ or $\bar{t} b$, but some exotic decay channels are open. The branching ratio of the decay $H^{ \pm} \rightarrow W^{ \pm} h_{1}$ and $H^{ \pm} \rightarrow W^{ \pm} a_{1}$ can maximally reach to $20 \%$ and $11 \%$, respectively, which can be used to distinguish the NMSSM from MSSM.

Because of the large SM backgrounds for the decay channel $H^{+} \rightarrow t \bar{b}$ and rather small decay branching ratio of the decay channel $H^{ \pm} \rightarrow \tau^{ \pm} \nu$, no evidence of a charged Higgs boson

is found at the $\mathrm{LHC}$ with $\mathcal{L}=36.1 \mathrm{fb}^{-1}$ integrated luminosity. The future higher luminosity and/or higher energy colliders would be useful for the detection of charged Higgs bosons. 


\section{Acknowledgement}

We thank Prof.Junjie Cao, Dr.Liangliang Shang and Dr.Yang Zhang for helpful discussions. This work was supported in part by the National Natural Science Foundation of China (NNSFC) under grant No.11705048, and the Program for Innovative Research Team in University of Henan Province under grant No.19IRTSTHN018.

[1] G. Aad et al. [ATLAS Collaboration], Phys. Lett. B 716, 1 (2012).

[2] S. Chatrchyan et al. [CMS Collaboration], Phys. Lett. B 716, 30 (2012).

[3] A. Djouadi, Phys. Rept. 459, 1 (2008) hep-ph/0503173.

[4] J. Cao, Z. Heng, T. Liu and J. M. Yang, Phys. Lett. B 703, 462 (2011) arXiv:1103.0631 [hep-ph]].

[5] J. Cao, Z. Heng, D. Li and J. M. Yang, Phys. Lett. B 710, 665 (2012) arXiv:1112.4391 [hep-ph]].

[6] J. Cao, Z. Heng, J. M. Yang and J. Zhu, JHEP 1210, 079 (2012) arXiv:1207.3698 [hep-ph]].

[7] J. Cao, Z. Heng, L. Shang, P. Wan and J. M. Yang, JHEP 1304, 134 (2013).

[8] U. Ellwanger, C. Hugonie and A. M. Teixeira, Phys. Rept. 496 (2010) 1 arXiv:0910.1785].

[9] J. J. Cao, Z. X. Heng, J. M. Yang, Y. M. Zhang and J. Y. Zhu, JHEP 1203, 086 (2012).

[10] Z. Heng, X. Gong and H. Zhou, Chin. Phys. C 42, no. 7, 073103 (2018).

[11] J. Cao, Y. He, L. Shang, Y. Zhang and P. Zhu, arXiv:1810.09143 [hep-ph].

[12] J. Cao, Y. He, L. Shang, W. Su and Y. Zhang, JHEP 1608, 037 (2016).

[13] J. Cao, Y. He, L. Shang, W. Su, P. Wu and Y. Zhang, JHEP 1610, 136 (2016).

[14] J. Cao, X. Guo, Y. He, P. Wu and Y. Zhang, Phys. Rev. D 95, no. 11, 116001 (2017).

[15] J. Cao, D. Li, L. Shang, P. Wu and Y. Zhang, JHEP 1412, 026 (2014).

[16] J. Cao, F. Ding, C. Han, J. M. Yang and J. Zhu, JHEP 1311, 018 (2013).

[17] N. D. Christensen, T. Han, Z. Liu and S. Su, JHEP 1308, 019 (2013).

[18] Z. Kang, J. Li and T. Li, JHEP 1211, 024 (2012) [arXiv:1201.5305 [hep-ph]].

[19] U. Ellwanger, JHEP 1203, 044 (2012) [arXiv:1112.3548 [hep-ph]];

[20] S. F. King, M. Muhlleitner and R. Nevzorov, Nucl. Phys. B 860, 207 (2012) arXiv:1201.2671 [hep-ph]]. 
[21] M. Guchait and A. H. Vijay, Phys. Rev. D 98, no. 11, 115028 (2018) arXiv:1806.01317 [hep$\mathrm{ph}]]$.

[22] Y. Hicyilmaz, L. Selbuz, L. Solmaz and C. S. Un, Phys. Rev. D 97, no. 11, 115041 (2018) arXiv:1711.07967 [hep-ph]].

[23] J. Y. Cen, J. H. Chen, X. G. He, G. Li, J. Y. Su and W. Wang, arXiv:1811.00910 [hep-ph].

[24] A. G. Akeroyd, S. Moretti and M. Song, Phys. Rev. D 98, no. 11, 115024 (2018) arXiv:1810.05403 [hep-ph]].

[25] A. Arhrib, R. Benbrik, H. Harouiz, S. Moretti and A. Rouchad, arXiv:1810.09106 [hep-ph].

[26] M. Aaboud et al. [ATLAS Collaboration], JHEP 1809, 139 (2018) arXiv:1807.07915 [hep-ex]].

[27] CMS Collaboration, CMS-PAS-HIG-18-014.

[28] M. Aaboud et al. [ATLAS Collaboration], JHEP 1811, 085 (2018) arXiv:1808.03599 [hep-ex]].

[29] B. Coleppa, F. Kling and S. Su, JHEP 1409, 161 (2014).

[30] T. Li and S. Su, JHEP 1511, 068 (2015).

[31] F. Kling, J. M. No and S. Su, JHEP 1609, 093 (2016).

[32] B. Coleppa, F. Kling and S. Su, JHEP 1412, 148 (2014);

[33] F. Kling, A. Pyarelal and S. Su, JHEP 1511, 051 (2015).

[34] M. Guchait and A. H. Vijay, Phys. Rev. D 98, no. 11, 115028 (2018) arXiv:1806.01317 [hep$\mathrm{ph}]$.

[35] U. Ellwanger, J. F. Gunion and C. Hugonie, JHEP 0502, 066 (2005) hep-ph/0406215;

[36] U. Ellwanger and C. Hugonie, Comput. Phys. Commun. 175, 290 (2006) hep-ph/0508022.

[37] D. J. Miller, R. Nevzorov and P. M. Zerwas, Nucl. Phys. B 681, 3 (2004) hep-ph/0304049].

[38] M. Drees, Int. J. Mod. Phys. A 4, 3635 (1989).

[39] S. F. King and P. L. White, Phys. Rev. D 53, 4049 (1996) hep-ph/9508346.

[40] P. A. R. Ade et al. [Planck Collaboration], Astron. Astrophys. 594, A13 (2016) arXiv:1502.01589 [astro-ph.CO]].

[41] M. Badziak, M. Olechowski and P. Szczerbiak, JHEP 1603, 179 (2016) arXiv:1512.02472 [hep-ph]].

[42] M. Badziak, M. Olechowski and P. Szczerbiak, JHEP 1707, 050 (2017) arXiv:1705.00227 [hep-ph]].

[43] P. Bechtle, O. Brein, S. Heinemeyer, G. Weiglein and K. E. Williams, Comput. Phys. Commun. 181, 138 (2010) arXiv:0811.4169 [hep-ph]]; 
[44] P. Bechtle, O. Brein, S. Heinemeyer, G. Weiglein and K. E. Williams, Comput. Phys. Commun. 182, 2605 (2011) [arXiv:1102.1898 [hep-ph]].

[45] P. Bechtle, S. Heinemeyer, O. Stal, T. Stefaniak and G. Weiglein, Eur. Phys. J. C 74 (2014) no.2, 2711 [arXiv:1305.1933 [hep-ph]].

[46] P. Bechtle, S. Heinemeyer, O. Stal, T. Stefaniak and G. Weiglein, JHEP 1411, 039 (2014) arXiv:1403.1582 [hep-ph]].

[47] O. Stal and T. Stefaniak, PoS EPS -HEP2013, 314 (2013) arXiv:1310.4039 [hep-ph]].

[48] L. Wang, J. M. Yang, M. Zhang and Y. Zhang, Phys. Lett. B 788, 519 (2019) arXiv:1809.05857 [hep-ph]].

[49] J. Alwall et al., JHEP 1407, 079 (2014) arXiv:1405.0301 [hep-ph]].

[50] P. Torrielli and S. Frixione, JHEP 1004, (2010) 110.

[51] D. Dercks, N. Desai, J. S. Kim, K. Rolbiecki, J. Tattersall and T. Weber, Comput. Phys. Commun. 221, (2017) 383.

[52] A. Aboubrahim and P. Nath, Phys. Rev. D 98, no. 9, 095024 (2018) arXiv:1810.12868 [hep$\mathrm{ph}]$.

[53] F. Kling, H. Li, A. Pyarelal, H. Song and S. Su, arXiv:1812.01633 [hep-ph].

[54] M. Cepeda et al. [Physics of the HL-LHC Working Group], arXiv:1902.00134 [hep-ph]. 